\title{
A CROSS-LAYER DESIGN APPROACH TO ENHANCE 802.15.4
}

\author{
Panagiotis Papadimitratos, Amitabh Mishra \\ Electrical and Computer Engineering, Virginia Tech \\ Blacksburg, VA \\ \{papadp, mishra\}@vt.edu
}

\begin{abstract}
The low-power communication in wireless sensor networks can be impacted by severe channel impairments. In this paper, to address this problem and achieve high network goodput, we propose that the medium access control protocol takes into consideration the error performance of the underlying wireless links. We combine a distributed backoff strategy regulated by the wireless link quality with Carrier Sense Multiple Access with Collision Avoidance. We integrate our cross-layer operational approach in the IEEE 802.15.4 standard, taking advantage of existing functionality and signaling to avoid network overhead and achieve simplicity in implementation. Our performance evaluation indicates that our scheme is more effective, achieving up to $69 \%$ higher goodput, and more efficient, delivering up to $154 \%$ more data bits per unit of energy consumed in the network, at the expense of an up to $18 \%$ degradation in fairness, compared to the basic 802.15.4.
\end{abstract}

\section{INTRODUCTION}

A wide range of emerging applications for wireless infrastructure-less networks call for low-complexity and lowcost enabling technologies, which rely on platforms with limited processing power and energy storage, capability for low-rate and short-range wireless communication only, and, overall, energy-efficient operation. Sensor and personal area networking, envisioned to enable scientific data collection, environmental and tactical monitoring, networking of portable devices, as well as devices embedded in physical environments, are prominent examples.

The development of a data link technology tailored to the requirements of such resource-constrained environments is paramount. The recently proposed IEEE 802.15.4 standard [2] was designed with such considerations in mind. It specifies a medium access control (MAC) and a physical layer $(P H Y)$ protocol, along with a set of capabilities for network organization. Information on the underlying channel is readily available through a pair of metrics: the Link Quality Indicator (LQI) and Energy Detection (ED), which can be provided by the 802.15.4 PHY along with each received frame. The 802.15.4 utilizes this information solely for configuration tasks, such as channel selection for a new coordinator-based personal area network. Nevertheless, a quantitative description of the wireless links' attributes, and in particular the channel's quality, can

This work is supported by the Department of Defense under contract no. H98230-04-P-2947/0000. and

Dwayne Rosenburgh

US Department of Defense

Fort Meade, MD

d. rosenburgh@ieee.org

be valuable at protocols above the $P H Y$. Such a cross-layer interaction can enhance the system performance by enabling the adaptation of the networking protocols [1]. This is especially true if cross-layer information is used to dynamically adapt the protocols' operation, rather than merely performing static network configuration tasks.

We take this cross-layer approach here, to enhance the 802.15.4 MAC protocol and improve the data link goodput. Despite the low duty-cycle operation, achieving data rates that approach the capacity of the wireless links is important in sensor networks. Events triggering measurements, and thus the activation of the nodes' networking modules, can generate a volume of information to be transported within a short period of time towards a network gateway (or sink). It is clearly desirable to have medium access control protocols that can effectively respond to such activity, being capable to handle high network load without relying on preconfigured communication. At the same time, the protocols must be robust to network impairments, which, in sensor networks can be significant [15], with frame loss rates of $20 \%$ or $30 \%$ [16].

In other words, in sensor networks, the performance of the data link layer is strongly dependent on the quality of the wireless link, in addition to the network load. Thus, not only does a $M A C$ protocol have to successfully balance between high utilization (collisions) and successful capturing of the medium (idle periods), as is true in any networking environment, but also take into consideration the underlying wireless channel errors.

This is exactly our approach in this paper, proposing a medium access control protocol that relies on two basic ideas and objectives: $(i)$ data flows with favorable channel conditions, that is, wireless links not suffering communication impairments, should be more likely to capture the medium, and (ii) error-prone data flows should be essentially 'separated' and thus be less likely to collide with successful transmissions. As a result, with fewer frames lost, the network undertakes fewer corrective actions (retransmissions), and higher overall communication rates can be achieved. Moreover, we are after a distributed and thus generally applicable protocol.

We propose the use of a Carrier Sense Multiple Access algorithm with Collision Avoidance (CSMA/CA) that determines the back-off period for each transmitting node based on the node's wireless link quality. We denote the quality of a $\left(u_{i}, v_{i}\right)$ link, or the link's error performance, by $c_{i}$. Then, the lower (better) $c_{i}$ is, the shorter the back-off period selected by $u_{i}$ should be, and vice versa. However, 
determining the appropriate back-off strategy is not an easy problem. Clearly, 'selecting' a transmission with favorable channel conditions reduces the likelihood of impairment. But transmissions may be deferred for relatively long periods of time when $c_{i}$ is high (worse). In other words, the back-off strategy must be balanced, between an approach that seeks to always select the 'best' channel conditions (and thus possibly suffer delays when such a link is not available), and an approach that seeks to maintain a high channel utilization (and thus possibly suffer loss of frames transmitted under unfavorable conditions).

We integrate our solution with the IEEE 802.15.4 MAC protocol, extending it towards our Cross Layer Design802.15.4 or CLD-802.15.4. Utilizing the 802.15.4 functionality and signaling to obtain, for example, the wireless link quality at the $M A C$ layer, offers the advantage of compatibility with the emerging de facto standard for personal area and sensor networking.

The performance evaluation results show that the $C L D$ 802.15.4 goodput is up to $69 \%$ higher and the energy efficiency is up to $154 \%$ higher compared to the basic 802.15.4, while the data delay of $C L D$-802.15.4 remains lower or equal to that of the basic 802.15.4. Nevertheless, the fairness among nodes with different link qualities can be up to $18 \%$ for $C L D-802.15 .4$. This being, admittedly, the cost of our scheme, we note that in sensor networks the overall system performance is more important than the performance of individual nodes.

In the rest of the paper, we first give an overview of the 802.15.4 medium access control protocol, followed by the presentation of our enhancement. Performance evaluation results are presented next. A survey of related state-of-theart and comparison to our approach is provided then, before a discussion of future work and our conclusions.

\subsubsection{OVERVIEW}

802.15.4 provides two approaches for medium access control: $(a)$ a coordinator-based $M A C$, with the coordinator providing synchronization and the structure of the channel access, with other nodes in its vicinity communicating through the coordinator, and $(b)$ a distributed $M A C$ with nodes communicating in a peer-to-peer manner. In the former case, the coordinator can provide guaranteed, contention-free access to a subset of nodes, or enable contentionbased access aligned with the boundaries of time slots, as nodes are synchronized (slotted access). In the latter case, only contention-based access is possible, but with no need for synchronization (unslotted access). Unslotted medium access is our focus in this paper, as peer-to-peer communication is generally applicable.

802.15.4 is based on the CSMA/CA algorithm shown in Figure $1{ }^{1} N B$ denotes the back-off stage or the number of

\footnotetext{
${ }^{1}$ Beyond the standard, 802.15.4 discussions can be found in [17], [18].
}

back-offs, and $B E$ is the back-off exponent, incremented after each back-off, so that the back-off value at the $j$-th stage is selected from an interval $\left[0, W_{j-1}\right]$, with $W_{j}=\left\{\begin{array}{ll}2^{\text {macMinBE }+j}, & 0 \leq j \leq B^{\prime} \\ 2^{\text {aMaxBE}}, & B^{\prime}<j \leq B\end{array}\right.$, parameters $\operatorname{aMaxBE}$ and $\operatorname{macMin} B E$ given in the list of protocol parameter values in Table 1 , and $B^{\prime}=a M a x B E-\operatorname{macMin} B E+1$. The time unit for the back-off counter is aUnitBackoffPeriod, a PHY-specific constant. ${ }^{2}$ The algorithm back-offs further if a Clear Channel Assessment (CCA) detects the medium to be busy. Once $N B$ reaches a protocol-specific maximum value, the $M A C$ protocol declares a transmission failure.

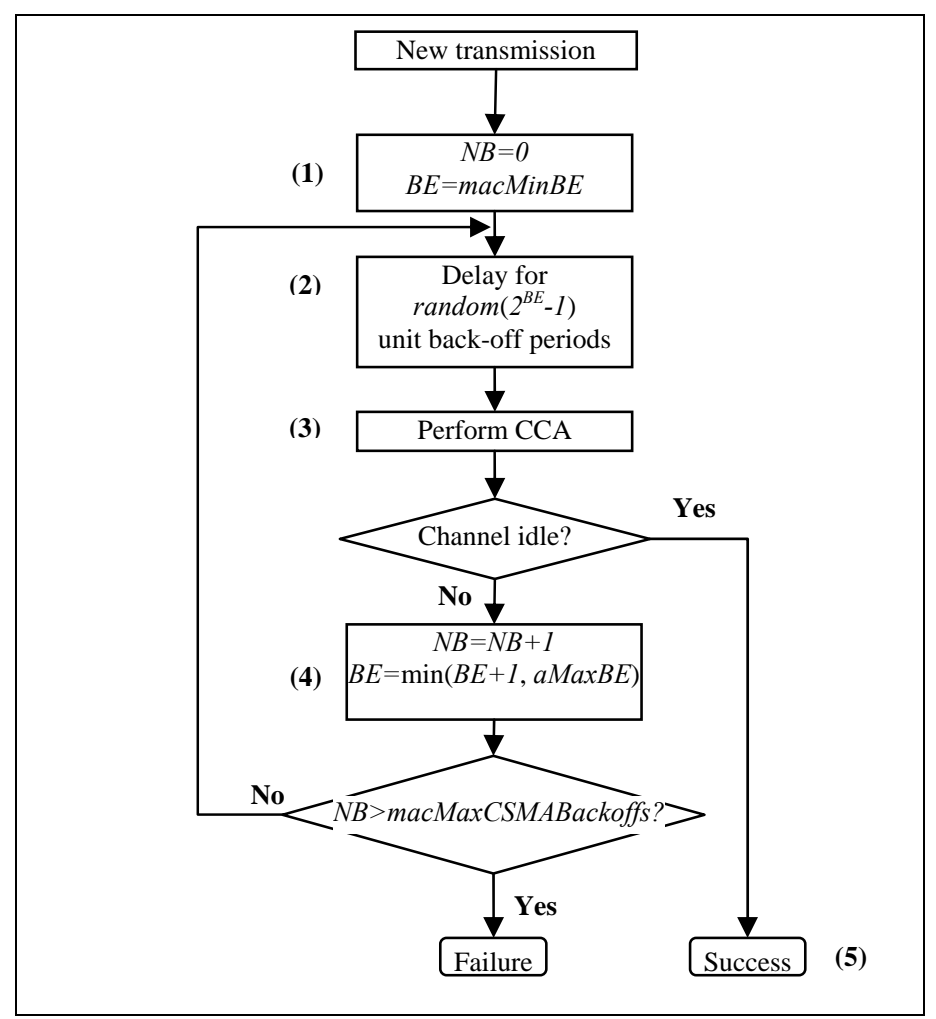

Figure 1. 802.15.4 CSMA/CA Algorithm (unslotted).

Figure 2 illustrates a frame transmission. After an initial short back-off period, the Collision Avoidance phase, the sender performs a $C C A$ for a period of time equal to $t_{C C A}$. The channel is found busy; after two additional backoffs the node finds the medium idle and transmits. In this example, frames are acknowledged; $t_{A C K}>$ aTurnaroundTime to allow reception of the $A C K$ at the sender. Moreover, successive frame transmissions are separated by an Inter-Frame Spacing (IFS), which, however, is not the spacing between any two successive frames on the channel; IFS solely ensures that the receiver has sufficient time to process frames [22].

\footnotetext{
2 The time periods are given in Symbols: the $868 / 915 \mathrm{MHz}$ bands' $P H Y$ symbols are bits, with duration of 0.05 and $0.025 \mathrm{msec}$ for data rates of 20 and $40 \mathrm{kbps}$ respectively, and $0.016 \mathrm{msec} / \mathrm{symbol}$ for the 2.4 $\mathrm{GHz}$ band $\mathrm{PHY}$ (4 bits/symbol and data rate of $250 \mathrm{kbps}$ ).
} 


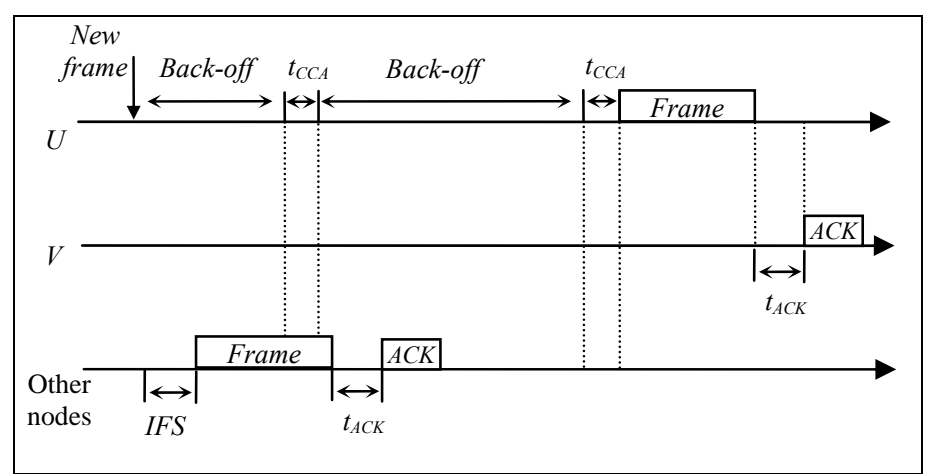

Figure 2. 802.15.4 Medium Access Data Transmission.

\begin{tabular}{|c|c|c|}
\hline Parameter & Value & Units \\
\hline aUnitBackoffPeriod & 20 & Symbols \\
\hline aMaxBE & 5 & - \\
\hline macMaxCSMABackoffs & 0 to 5 (Default 4) & - \\
\hline macMinBE & 0 to 3 (Default 3) & - \\
\hline aMaxFrameOverhead & 25 & Bytes \\
\hline aMaxPHYPacketSize & 127 & Bytes \\
\hline aMaxMACFrameSize & 102 & Bytes \\
\hline aMaxFrameRetries & 3 & - \\
\hline aMaxBeaconOverhead & 75 & Bytes \\
\hline aMinLIFSPeriod & 40 & Symbols \\
\hline aMinSIFSPeriod & 12 & Symbols \\
\hline aTurnaroundTime & 12 & Symbols \\
\hline macAckWaitDuration & 54 to 120 (Default 120) & Symbols \\
\hline$t_{\text {CCA }}$ & 8 & Symbols \\
\hline
\end{tabular}

Table 1. 802.15.4 Parameters.

\section{CLD-802.15.4}

$C L D$-802.15.4 requires the calculation of the $c_{i}$ estimates, and, essentially, the modification only of Steps (2) and (4) of the algorithm shown in Figure 1, with the operation of the protocol otherwise following that of the basic 802.15.4. Each node $u_{i}$ calculates an estimate of the $\left(u_{i}, v_{i}\right)$ wireless link error performance or Bit Error Rate $(B E R)$ denoted by $c_{i}$; the higher $c_{i}$ is, the more likely it is that a frame be impaired by the medium. With an up-to-date estimate of $c_{i}$ at hand, $u_{i}$ selects a back-off interval $b_{i}$ as a function of $c_{i}$. The core characteristic of $C L D-802.15 .4$ is the selection of $b_{i}$ : for two nodes $u_{i}$ and $u_{j}$ with $c_{i} \leq c_{j}$, the back-off intervals must be such that $b_{i} \leq b_{j}$. This way, $u_{i}$ 's transmissions are more likely to precede those from $u_{j}$, which are thus less likely to obstruct (contend with) $u_{i}$.

\section{Back-off Selection}

We define $C=\left\{C_{1}, C_{2}, \ldots, C_{K}\right\}$ a set of disjoint intervals such that any of the estimated $c_{i}$ values falls in exactly one $C_{k} \in C$. $K$ is a design parameter of the protocol. Moreover, $m_{k}$ denotes the maximum number of back-offs for nodes in $C_{k} \in C$. If $0 \leq j \leq m_{k}$ is the index of the current back-off stage for a node $u_{i}$, then, $b_{i} \sim F_{B}\left(b ; h\left(C_{k}, j\right)\right)$ is randomly drawn from a distribution $F_{B}($ ) with parameter $h\left(C_{k}, j\right)$ for nodes at stage $j$ with $c_{i} \in C_{k}$. $F_{B}()$ is uniform and $h\left(C_{k}, j\right)=\left[L_{k, j}, U_{k, j}\right]$ is a vector of the upper and lower limits of the range the back-off value is drawn from.

We design three heuristic variants of the back-off strategy, which we denote as $C L D_{1}, C L D_{2}$, and $C L D_{3}$. In all cases, all nodes have the same maximum number of backoff stages, $m=4$, the default 802.15 .4 value [2]. For $C L D_{1}$, $|C|=4$, while for $C L D_{2}$ and $C L D_{3}|C|=5$. The $x$-axes in Figure 3, show the $C_{k}$ intervals for these strategies, with the $y$-axes showing the initial (at stage $j=0$ ) back-off upper and lower limits for each $C_{k}$ interval.

The three strategies also differ in how their back-offs change as $j$ increases. With $\max U$ and $\max L$ protocol selectable constants, for $0 \leq j \leq m$ :

$\boldsymbol{C L} \boldsymbol{D}_{1}$ and $\boldsymbol{C} \boldsymbol{L} \boldsymbol{D}_{2}: L_{k, j}=L_{k, 0}$

$$
U_{k, j}=\min \left\{\left(U_{k, 0}+1\right) 2^{j}-1, \max U\right\}
$$

$C L D_{3}:$

$$
\begin{aligned}
& L_{k, j}=\min \left\{\left(L_{k, 0}+1\right) 2^{j}-1, \max L\right\} \\
& U_{k, j}=\min \left\{\left(U_{k, 0}+1\right) 2^{j}-1, \max U\right\}
\end{aligned}
$$

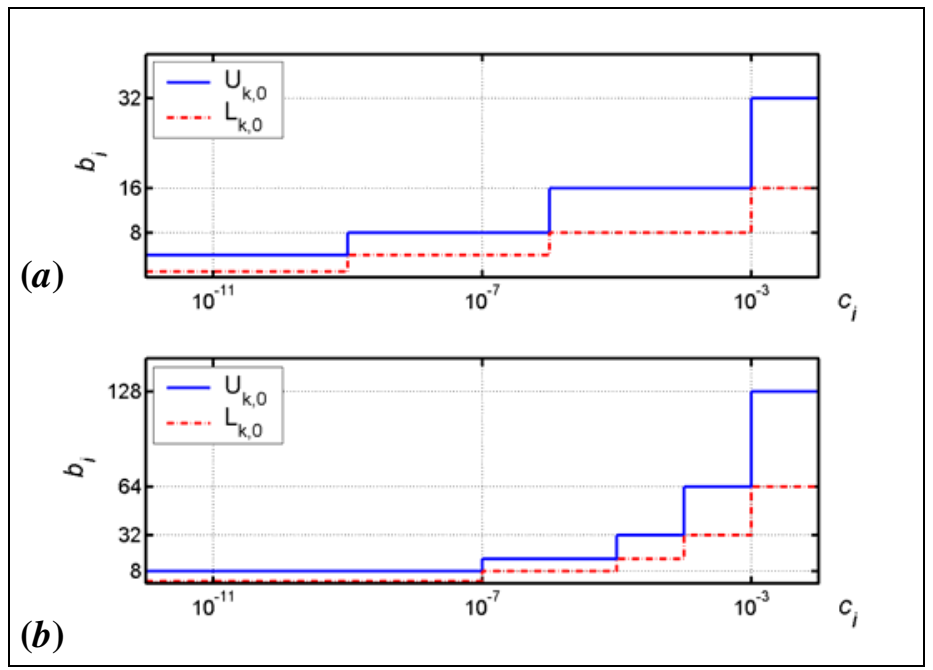

Figure 3. Back-off selection, initial back-off. (a) $C L D_{1}$, (b) $C L D_{2}$ and $C L D_{3}$.

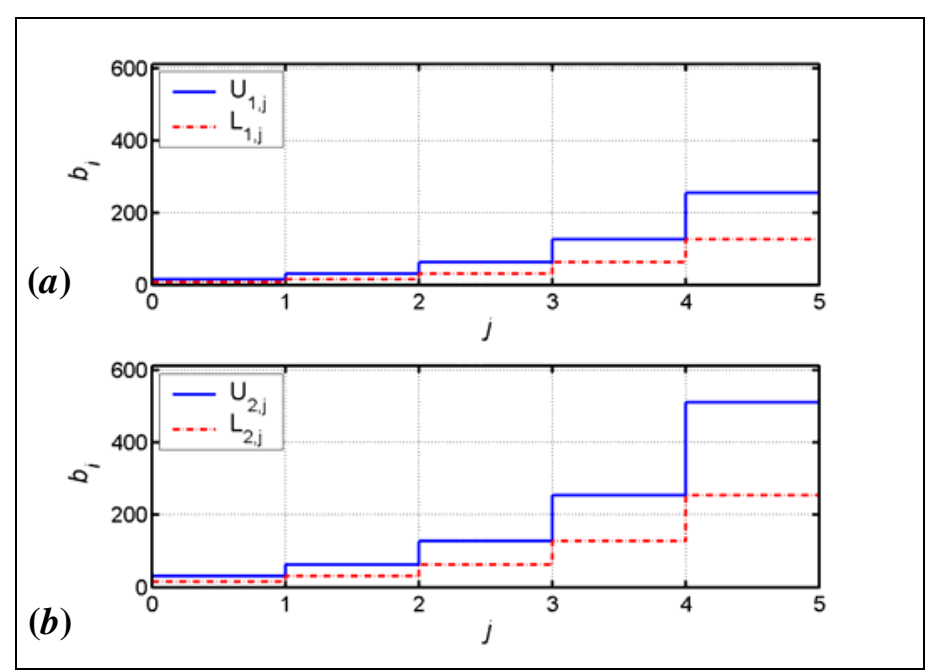

Figure 4. Back-off selection $\left(b_{i}\right)$ as a function of the back-off stage (j) for $C L D_{3}$ and nodes in: (a) $C_{1}$, (b) $C_{2}$. 
Figure 4 illustrates the back-off limits for $\mathrm{CLD}_{3}$ and nodes with $c_{i} \in C_{1}$ (Fig. 4.(a)) and $c_{i} \in C_{2}$ (Fig. 4.(b)), as a function of the back-off stage. Intuitively, in all three strategies, the initial back-off 'separates' nodes in different $C_{k}$ 's. But the degree this 'separation' is maintained at subsequent stages differs among the three strategies: $C L D_{3}$ ensures that senders with better $c_{i}$ values remain more likely to capture the medium even after successive failed transmission attempts and thus back-offs. We set $\max U$ to 31, 127, and 1023 for $C L D_{1}, C L D_{2}$, and $C L D_{3}$ respectively, and $\operatorname{maxL}$ to 511 for $C L D_{3}$.

\section{Optimal Back-off Strategy}

The back-off strategy is determined by the algorithm parameters $\underline{m}=\left[m_{k}\right]_{1 \times K}$ and $\underline{h}=\left[h\left(C_{k}, j_{i}\right)\right]_{M \times K \times 2}$, with $M=\max \left\{m_{k}\right\}$, and depends on the design choice of the $C=\left\{C_{1}, C_{2}, \ldots, C_{K}\right\}$ set. We define the optimal back-off strategy, for a given set $C$, to be the parameters $\underline{m}$ and $\underline{h}$ such that the average goodput, or the rate of successfully delivered data at the $M A C$ layer, $\bar{G}$ is:

$$
\arg \max _{\underline{m}, \underline{h}}\{\bar{G}\}
$$

We are interested in maximizing goodput rather than throughput, $S$, the rate of successfully transmitted data, exactly because transmission impairments and collisions are both significant sources of frame errors. If $P_{\text {ERROR }}$ is the probability of frame error, given that a single node captured the medium and transmitted, then, $G=S\left(1-P_{E R R O R}\right) .^{3}$

For $N$ active contending nodes, let $\underline{n}=\left[n_{1}, n_{2}, \ldots, n_{K}\right]$ be the vector of $n_{k} \geq 0$, not all zero simultaneously, the numbers of nodes with $c_{i} \in C_{k}$, with $\sum_{k=1}^{K} n_{k}=N$. Assuming that $c_{i} \sim F_{B E R}$ ( ) follows a distribution of link error performance, which depends on the signal-to-noise ratio, the modulation, and coding of the physical layer [19], [20], [21], we denote $\operatorname{Pr}\left\{c_{i} \in C_{k}\right\}=p_{k}$. Then, assuming that $c_{i}$ are independent, the probability of a link quality assignment $\underline{N}=\underline{n}$ is $\operatorname{Pr} \underline{\{N}=\underline{n}\}=\frac{n !}{n_{1} ! n_{2} ! . . n_{K} !} p_{1}^{n_{1}} p_{2}^{n_{2}} \ldots p_{K}^{n_{K}}$. Since $P_{\text {ERROR }}$ depends on the channel quality of the contending nodes, we maximize numerically, for a given $N$, the average goodput, $\bar{G}$, over all possible link quality assignments, $\underline{N}$.

\footnotetext{
${ }^{3}$ The derivation of analytical expressions of $S$, $G$, and average delay is the topic of another publication that analyzes the basic 802.15.4. The analysis of $C L D-802.15 .4$ and derivation of the optimal strategy will be presented in the journal version of this paper.
}

\section{Link Quality Estimation}

Nodes can calculate the $c_{i}$ link metrics in different ways. The sender can use feedback from the corresponding receiver, as 802.15.4 provides $A C K$ messages for each frame. Similar approaches to estimate the link error probability were taken in [7], [27], [28], and [29]. The sender can also calculate $c_{i}$ estimates upon receiving traffic from the intended receiver, assuming symmetry in the channel performance. Existing signaling can be utilized to avoid overhead: low duty-cycle operation and network configuration rely heavily on the (periodic) transmission of beacons (e.g., [3], [4]), $u_{i}$ can wait for a beacon transmission from $v_{i}$. In general, the $u_{i}$ to $v_{i}$ transmission will follow immediately the transmission of such a control message. A characteristic example is the case of the beacon-enabled 802.15.4, where the active part of $u_{i}$ 's duty cycle follows the beacon. Moreover, $A C K$ s themselves can serve to acquire $c_{i}$, especially if $u_{i}$ transmits bursts of frames (e.g., [3]). In all such cases, the Link Quality Indicator (LQI) and Energy Detection $(E D)$ measurements are provided for each received frame by the 802.15.4 PHY.

\section{PERFORMANCE EVALUATION}

Our performance evaluation shows that $C L D-802.15 .4$ outperforms basic 802.15.4, achieving up to $69 \%$ higher goodput, which is the objective of our design. Moreover, $C L D-802.15 .4$ is more efficient, imposing significantly lower load and delivering up to $154 \%$ more bits of information per energy unit than basic 802.15.4, with delay less than or equal to that of 802.15 .4 . These improvements come at the expense of a $7-18 \%$ decrease in the fairness among contending individual nodes, compared to the basic 802.15.4.

We consider the case of collocated contending nodes, all operating at same frequency band, with any two transmissions overlapping in time resulting in a collision at a receiver. Nodes are equipped with half-duplex radios [25], and at any point in time $N$ nodes contend for the medium. Nodes are not synchronized and, thus, transmissions, which are acknowledged, are not aligned with slot boundaries. All senders always have a packet to send and their duty cycle is set to $100 \%$. This, although not true in practice, allows us to isolate the performance of 802.15.4 and $C L D-802.15 .4$ as the dependence of $M A C$ delays on the idle/active periods, a mechanism beyond the $M A C$ itself, is removed; otherwise, delays for frame transmissions initiated close to the end of the active period (of the sender or the receiver) would be inflated by the length of the idle period. The results are also meaningful for cases when a subset of the collocated nodes has a packet ready for transmission. Note also that saturation conditions have been used in the evaluation of other $M A C$ protocols e.g., [23], [12]. Each sender transmits to the same receiver throughout the simulation, which is 5 seconds. Each point on the graphs is averaged over at least 100 randomly seeded runs, and $99 \%$ confidence intervals are shown. 


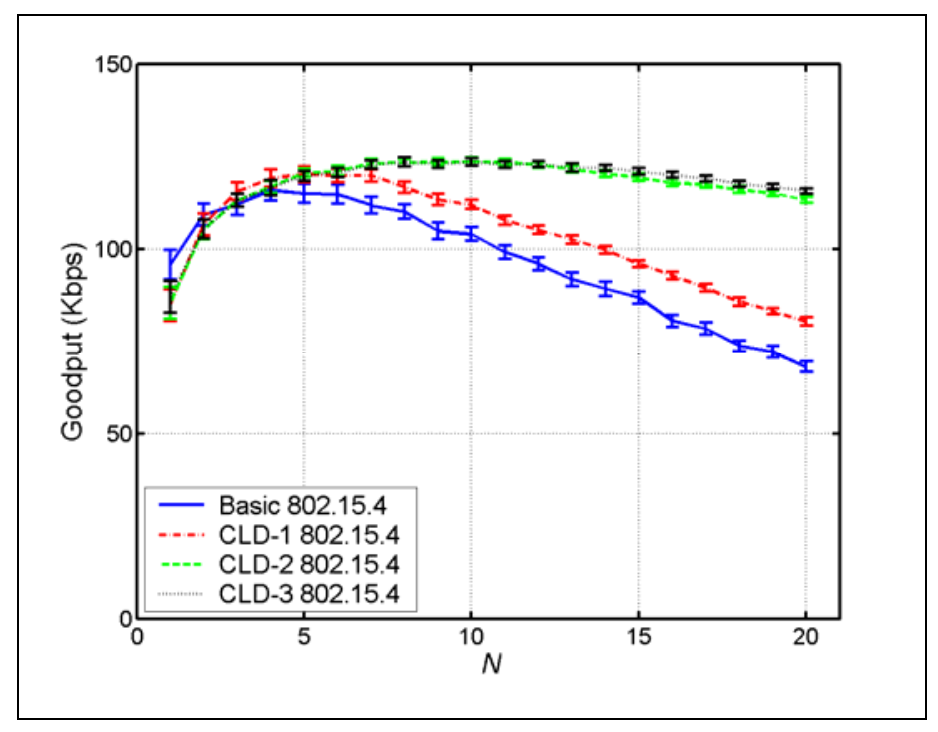

Figure 5. Goodput.

We evaluate all three $C L D_{1}, C L D_{2}$, and $C L D_{3}$ strategies and compare them to the basic 802.15.4. We use the default 802.15 .4 constant values [2], apart from the back-off interval parameters for $C L D_{2}$ and $C L D_{3}$ defined in the protocol operation section. The frame payload is $X=102$ bytes; we experimented with smaller $X$ values (e.g. 64 bytes), but we do not report the results here as we observed the same trends. Channel error conditions are randomly generated for each pair of communicating nodes with bit error rate values drawn uniformly randomly [30], from a $\left[10^{-12}, 10^{-2}\right]$ interval. We treat the events of a CSMA/CA ChannelAccessFailure (Figure 1, when NB > macMaxCSMABackoffs) and a transmission failure due to channel impairment (i.e., no $A C K$ received) as equivalent, as in either case a frame is retransmitted (up to aMaxFrameRetries times).

Figure 5 shows that all variants of $C L D$-802.15.4 outperform basic 802.15.4: $C L D_{1}, C L D_{2}$ and $C L D_{3}$ achieve up to $18 \%, 66 \%$ and $69 \%$ higher goodput respectively. The improvement of $C L D$-802.15.4 is significant for $N>4$ and grows as $N$ increases. Clearly, it is not meaningful for a single node to defer its transmission, and, similarly, with only a few nodes contending, the benefits of $C L D-802.15 .4$ are not realized. But as $N$ increases, it becomes increasingly likely that one or more flow experience favorable channel conditions, being more likely to capture the medium, and thus improve the overall performance.

A more detailed look reveals that $C L D$-802.15.4 reduces the numbers of frames corrupted by the channel up to $75 \%$ (Figure 6.(a)). Nevertheless, as the load increases (i.e., as $N$ increases), the $C L D$-802.15.4 reduction in the number of corrupted frames decreases slightly. A node with worse channel quality becomes more likely, in high load conditions, to capture the medium when a node with a better channel quality is at a higher back-off stage, as shown in Figure 4. This is so because the channel is found busy with higher probability, and nodes are more frequently at a higher back-off stage.

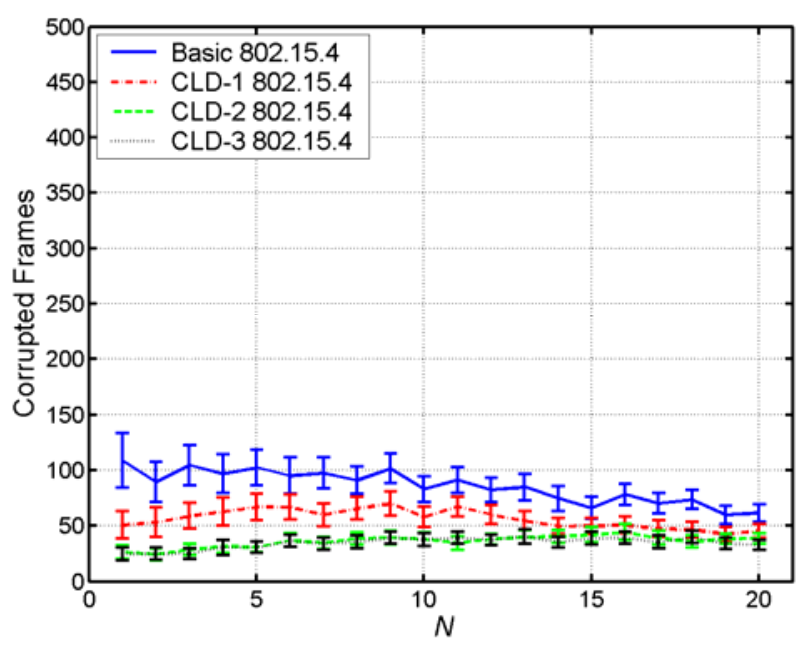

(a)

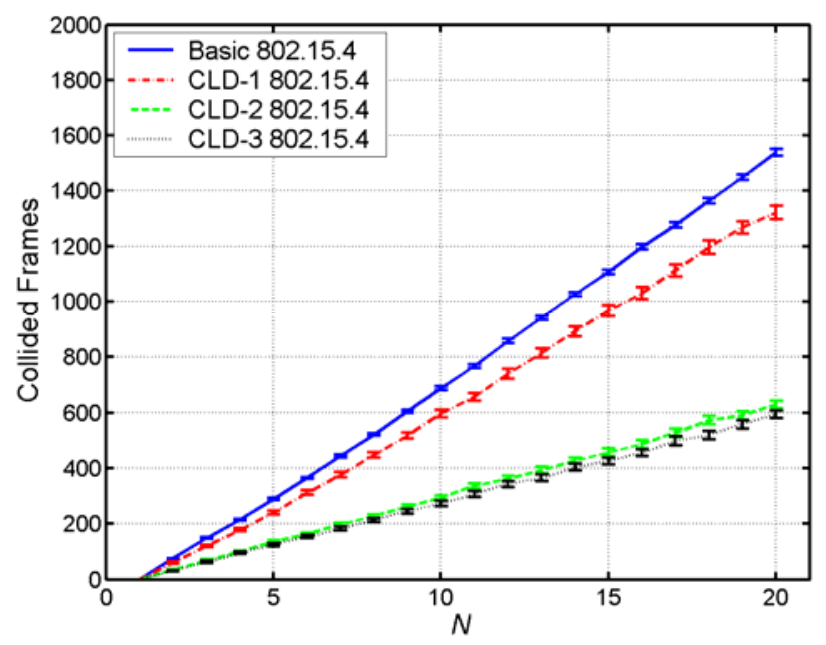

(b)

Figure 6. Numbers of frames (a) Impaired by the medium, (b) Involved in a collision.

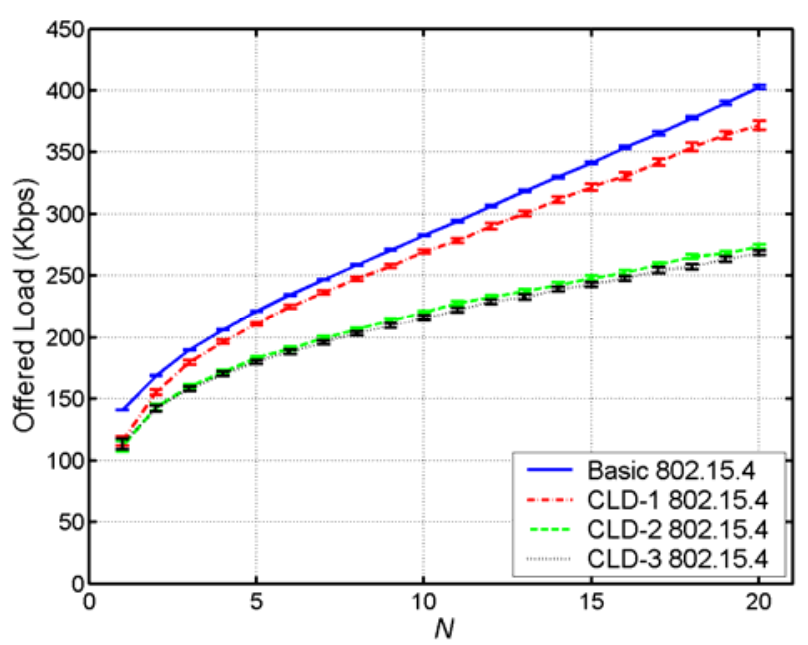


Figure 7. Load.

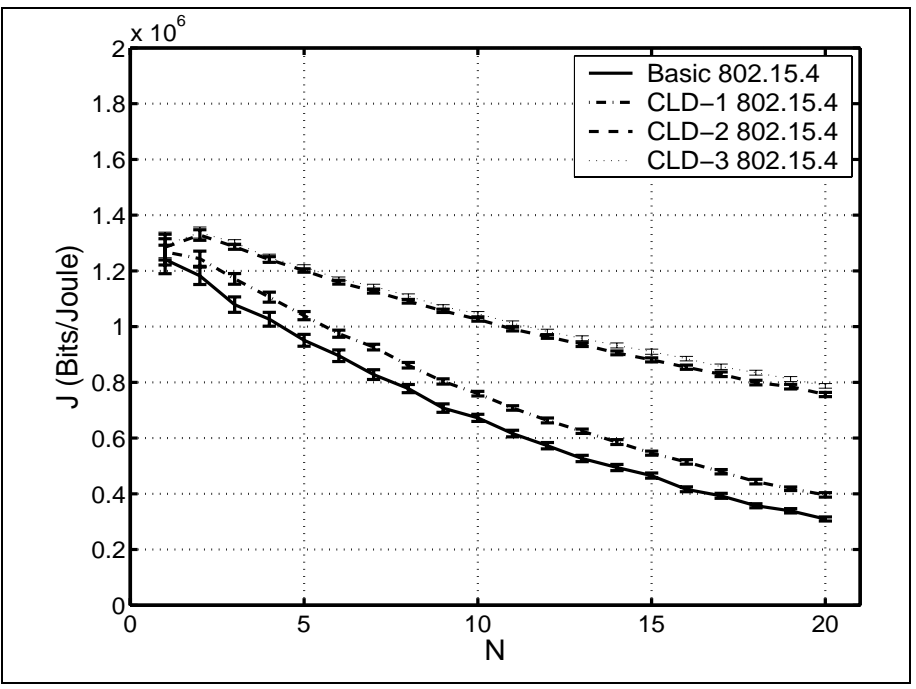

Figure 8. Energy Efficiency $(J)$, in (delivered) bits/joule.

The second factor of $C L D$-802.15.4 improvement over basic 802.15.4 is shown in Figure 6.(b): the back-off strategy is effective in 'separating' the contending transmissions and the number of collided frames decrease up to $146 \%$ respectively. The combined reduction of collisions and frame impairments renders retransmissions less frequent. This implies that the network overhead per delivered frame reduces significantly. As shown in Figure 7, the load for $C L D$-802.15.4 is lower and the number of delivered frames is up to $69 \%$ for $C L D-802.15 .4$.

The efficiency of $C L D-802.15 .4$ becomes more apparent in Figure 8, which shows the energy efficiency index $J$ in delivered bits/joule, calculated as the total number of delivered bits, divided by the total energy consumed at all nodes. $C L D-802.15 .4$ is up to $154 \%$ energy efficient than basic 802.15 .4 , exactly because it achieves higher reliability in communication with lower overhead. We provide a detailed energy consumption model in the Appendix.

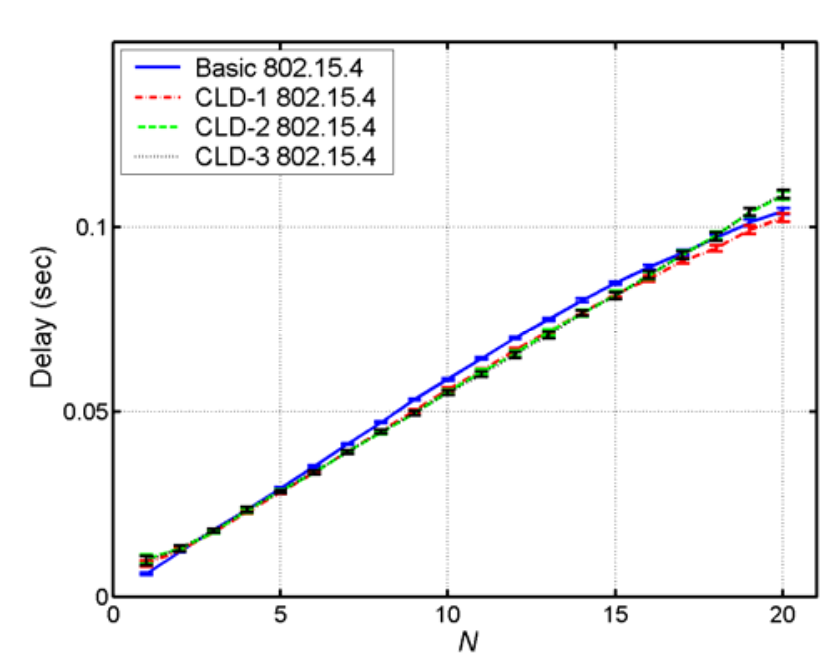

Figure 9. Average Delay.

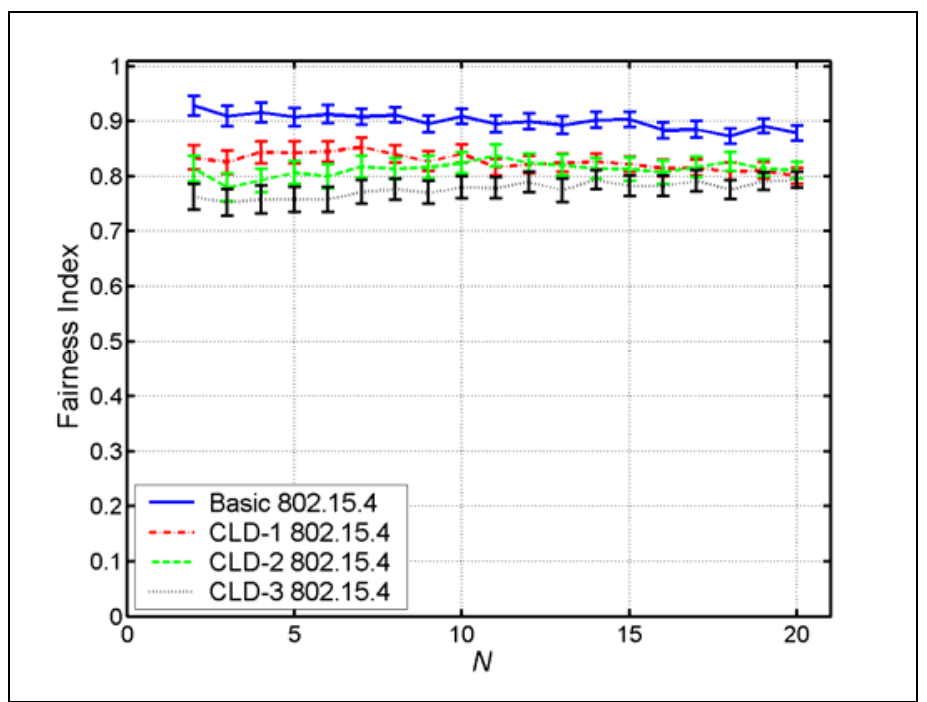

Figure 10. Fairness.

We also observe that the average data delay, in Figure 9, measured for delivered frames, is very similar for both the basic 802.15 .4 and $C L D$-802.15.4 (up to $7 \%$ lower). This is so, despite the possibly longer back-off periods for $C L D$ 802.15.4. However, this delay increase, due to longer backoffs, is somewhat counterbalanced by the relatively more frequent transmissions by nodes with favorable channel conditions and the up to $76 \%$ fewer retransmissions for CLD-802.15.4.

We calculate the fairness index [5], $F I=\left(\sum_{l=1}^{N} G_{l}\right)^{2} / N\left(\sum_{l=1}^{N} G_{l}^{2}\right)$, where $G_{l}$ is the goodput of each node. Figure 10 shows that the $F I$ for $C L D-802.15 .4$ is up to $18 \%$ lower than the $F I$ for the basic 802.15.4. Essentially, this is the cost of our scheme. More precisely, CLD802.15.4 trades-off the moderate fairness degradation for up to $69 \%$ improvement in goodput and $154 \%$ energy efficiency. We also observe that the relative improvement from $C L D_{2}$ to $C L D_{3}$ is at the order of $2-3 \%$, while the decrease in the $F I$ is up to $7 \%$. This suggests that no significant improvement could be achieved even if fairness was further sacrificed.

\section{RELATED WORK}

The use of channel state information has been used in different ways in prior literature. [9], [10] extend the Aloha protocol, providing methods so that each sensor node controls its transmission towards a sink (or access point). In [9], nodes can adjust their transmission power under a global constraint on the average consumed power. Then, the optimal probability is derived for nodes to transmit as long as the channel gain is above a threshold. Similarly, 
[10] assumes the knowledge of a threshold, but provides a more general treatment of the problem and the transmission control method, for Code Division Multiple Access (CDMA) systems and the simultaneous reception of multiple packets at the sink. Our work is different, investigating and extending a Carrier Sense Multiple Access (CSMA) protocol. Our motivation was two-fold: on the one hand, CSMA protocols were shown to achieve significantly higher throughput than slotted Aloha [26], and they were integrated in the 802.15.4 standard [2]. In this context, we propose the modification of the back-off algorithm to control transmissions based on the wireless link error performance, and address technical considerations specific to the 802.15.4 standard.

The back-off algorithm and its optimization was proposed in different contexts, but none of these works utilized information on the physical layer channel or considered the challenge of error-prone links in sensor networks. [12], [24], analyzed and proposed alternative p-persistent CSMA algorithms for 802.11 networks [11] adapting to the level of contention through an estimate of the number of contending nodes. A more recent work proposed Kalman filtering to estimate the number of contending nodes and improve performance of 802.11 networks [13]. Moreover, the 802.11 back-off algorithm was used to provide fairness and serve flows of different priorities [14], or optimized as a function of contending nodes [31].

Channel state information was used to determine the transmission data rate [6], [7], for 802.11-based networks, with those approaches entailing complexity inappropriate for sensor networks. The challenge lies in that 802.15.4 PHY does not provide the option for multi-rate transmissions. Different data rates are possible only in different bands, e.g., $250 \mathrm{Kbps}$ in the ISM band $(2.4 \mathrm{GHz})$ and $40 \mathrm{Kbps}$ in the $915 \mathrm{MHz}$ band. It is thus necessary, should nodes communicating at $40 \mathrm{Kbps}$ wish to increase their rate, to negotiate and select a new channel (band) before proceeding, clearly an approach particularly complex and impractical in the light of the considerations in [6], [7]. $M A C$ protocols proposed for sensor networks, such as [3], [8], have attractive features but do not use wireless link information to regulate medium access.

\section{DISCUSSION AND FUTURE WORK}

The accuracy of the link quality is an important parameter. Our protocol design depends on the interval $C_{k}$ those estimates belong to. As a result, the problem of accuracy of estimating network metrics, common all related schemes, is somewhat alleviated. For future work, we will evaluate the robustness of our protocol with inaccurate estimates, when $c_{i}$ falls outside its actual $C_{k}$ interval. Moreover, we will investigate the sensitivity of the protocol performance to the design of choice of $C$. Here, we determined the $C_{k} \in C$ intervals so that, for any two link quality values $c_{i}, c_{i}^{\prime}$ in neighboring $C_{k}, C_{l}$, the difference in the respective frame error rates is significant, i.e., $\left|\left(1-c_{i}\right)^{X+H}-\left(1-c_{i}^{\prime}\right)^{X+H}\right|>\Delta$, above protocol selectable constants $\Delta>0$, for $X$ and $H$ the frame payload and header sizes in bits.

The issue of fairness among nodes with different channel conditions was investigated in the performance evaluation. The results clue that the trade-off of fairness for improvements in performance cease to be significant beyond a certain point. Moreover, we conjecture that the moderate degradation of fairness is less important in sensor networks than other ad hoc networks, as the overall system (network) performance is more important that the level of service or share of the resources available to individual nodes. We intend to investigate this broad problem further, especially in conjunction with the routing layer operation.

\section{CONCLUSIONS}

We proposed an enhancement to the IEEE 802.15.4 standard, based on a $C S M A / C A$ algorithm that regulates access to the medium according to the underlying wireless channel quality. Our performance evaluation shows that the proposed scheme increases the goodput up to $69 \%$ and delivers up to $154 \%$ more bits per unit of energy consumed in the network, compared to the basic 802.15.4, at the expense of a moderate degradation of individual node fairness. As a result, we believe that, along with the compatibility with the de facto sensor networking standard, our scheme can yield highly efficient and robust communication.

\section{REFERENCES}

[1] Goldsmith, A., and Wicker, S., "Design Challenges for Energy-constrained Ad Hoc Wireless Networks," IEEE Wireless Communications, Vol.9, No.4, pp.8-27, Aug. 2002

[2] IEEE Std 802.15.4: Wireless Medium Access Control (MAC) and Physical Layer (PHY) Specifications for LowRate Wireless Personal Area Networks (LR-WPANs), May 2003

[3] Ye, W. Heidemann, J., and Estrin, D., "Medium Access Control with Coordinated Adaptive sleeping for wireless sensor networks," IEEE Trans. on Networking, Vol.12, No.3, Jun 2004

[4] Schurgers, C. et al., "Optimizing Sensor Networks in the Energy-Latency-Density Design Space," IEEE Trans. Mob. Comp., Vol.1, No. 1, Jan. 2002

[5] Jain, R., et al., "Fairness, Call Establishment Latency and Other Performance Metrics,” TR 96-1173, ATM Forum, Aug. 1996

[6] Sadeghi, B., Kanodia, V., Sabharwal, A., and Knightly, E., "Opportunistic Media Access for Multirate Ad Hoc Networks," in proceedings of the $8^{\text {th }}$ ACM MobiCom, Atlanta, GA, Sept. 2002

[7] Holland, G., Vaidya, N., and Bahl, P., "A Rate-Adaptive MAC Protocol for Multihop Wireless Networks," in proceedings of the $7^{\text {th }} A C M$ MobiCom, Rome, Italy, Sept. 2001 
[8] Polastre, J., Hill, J., Culler D., "Versatile Low Power Media Access for Wireless Sensor Networks," in proceedings of SenSys'04, Baltimore, MD, Nov. 2004.

[9] Qin, X., and Berry, R., "Exploiting Multiuser Diversity for Medium Access Control in Wireless Networks," in proceedings of INFOCOM 2003, San Francisco, CA, Mar. 2003

[10] Venkitasubramanian, P., Adireddy, S., and Tong. L., "Sensor Networks with Mobile Agents, Optimal Random Access and Coding,” IEEE JSAC, Vol. 22, No.6, Aug. 2004

[11] IEEE Std 802.11: Wireless LAN Medium Access Control (MAC) and Physical Layer (PHY) Specification, Nov. 1997

[12] Cali, F., Conti, M., and Gregori, E., "Dynamic Tuning of the IEEE 802.11 Protocol," IEEE/ACM Transactions on Networking, Vol. 8, No. 6. pp. 785-799, Dec. 2000

[13] Bianchi, G., and Tinnirello, I., "Kalman Filter Estimation of the Number of Competing Terminals in an IEEE 802.11 Network,” in proceedings of the IEEE Infocom, Apr. 2003

[14] Vaidya, N., Bahl, P., and Gupta, S., "Distributed Fair Queueing in a Wireless LAN,” in proceedings of the ACM Mobicom '00, Boston, MA, Aug. 2000

[15] Woo, A., Tong, T., and Culler, D., "Taming the underlying challenges of reliable multihop routing in sensor networks," in proceedings of the SenSys 2003, Los Angeles, CA, Nov. 2003

[16] Wan, C., Campbell, A., and Krishnahmurthy, L., "PSFQ: A Reliable Transport Mechanism for Wireless Sensor Networks.” in proceedings of the ACM International Workshop on Wireless Sensor Networks and Applications, Atlanta, GA, Sept 2002

[17] Callaway, E., Gorday, P., Hester, L., Gutierrez, J.A., Naeve, M., Heile, B., and Bahl, V., "Home networking with IEEE 802.15.4: a developing standard for low-rate wireless personal area networks," IEEE Communications Magazine, Vol. 40, No. 8, pages 70-77, Aug. 2002

[18] Gutierrez, J.A., Naeve, M., Callaway, E., Bourgeois, M., Mitter, V., and Heile, B., "IEEE 802.15.4: a developing standard for low-power low-cost wireless personal area networks," IEEE Network, Vol. 15, No. 5, pages 12-19, Sept.-Oct. 2001

[19] Simon, M., and Alouini, M-S., “A Unified Approach to the Performance Analysis of Digital Communication over Generalized Fading Channels," Proceedings of the IEEE, Vol. 86, No. 9, Sept. 1998

[20] Proakis, J.G., Digital Communications, 3rd edition, McGraw-Hill, 1995

[21] Wang, H., and Moayeri, N., "Finite State Markov Channel: A Useful Model for Radio Communication Channels," IEEE Trans. of Vehicular Tech., Vol. 44, no. 1, Feb. 1995

[22] Seward, D., Callaway, E., and Cragie, R., Personal Communication, IEEE WLAN TG4b mailing list, Mar. 2005

[23] Bianchi, G., "Performance Analysis of the IEEE 802.11 Distributed Coordination Function," IEEE Journal on Selected Areas in Communications, Vol. 18, No. 3, Mar. 2000

[24] Cali, F., Conti, M., Gregori, E., “IEEE 802.11 Protocol : Design and Performance Evaluation of an Adaptive Backoff Mechanism,” IEEE JSAC, Vol. 18, No. 9, Sept. 2000

[25] MICAz radio and processing board - URL: http://www.xbow.com/Products/Product_pdf_files/Wireless _pdf/MICAz_Datasheet.pdf
[26] Kleinrock, L., and Tobagi, F., "Packet Switching in Radio Channels: Part I - Carrier Sense Multiple-Access Modes and Their Throughput-Delay Characteristics," IEEE Transactions, Vol. COM-23, No. 12, Dec. 1975

[27] IEEE 802.11h Standard, "Part 11, Amendment 5: Spectrum and transmit power management extensions in the $5 \mathrm{GHz}$ band in Europe,” 2003

[28] Keshavarzin, A., Uysal-Biyikoglou, .E, Hermann, F., and Manjeshwar, A., "Energy-efficient link assessment in wireless sensor networks, " in proceedings of the IEEE Infocom 2004, Mar. 2004

[29] Kopke, A., Willing, A., and Karl, H., "Chaotic maps as parsimonious bit error models of wireless channels," in proceedings of the of the IEEE Infocom, Apr. 2003

[30] Lee, S., Bhattacharjee, B., and Banerjee, S., "Efficient Geographic Routing in Multihop Wireless Networks,” in the proceedings of Mobihoc 2005, Urbana-Champagne, IL, May 2005

[31] Haas, Z. J. and Deng, J., "On Optimizing the Back-off Interval for Random Access Schemes,” IEEE Transactions on Communications, Vol. 51, No. 12, Dec. 2003

\section{APPENDIX}

We consider $M I C A z$ radios [25], operating in the $2.4 \mathrm{GHz}$ band at $250 \mathrm{Kbps}$, with transmission power level at $0 \mathrm{dBm}$, outdoor typical range of 75 meters, transceiver start-up time approximately $1.5 \mathrm{~ms}$, and $3 \mathrm{~V}$ power supply. For those radios, the current draw for reception (receiver on) is $19.7 \mathrm{~mA}$, the current draw is $20 \mu \mathrm{A}$ when the receiver is off, and the current for transmission is $17.4 m A$.

The receiver can be turned off while the node does not receive, transmit, or perform $C C A$ to preserve energy. This way, prolonged back-off periods, which are in general longer for $C L D$ 802.15 .4 , will not increase power consumption. This is especially so, as nodes do not need to listen to the medium (unlike 802.11 for example) while backing off. Care is nonetheless taken to allow for sufficient start up time, $T_{\text {start-up }}$; when the back-off interval is less than 8 time units (aUnitBackoffPeriod, in Table 1), the receiver remains on, and otherwise it is powered off.

The following two equations model the energy cost of a successful (Eq. A.1) and failed (Eq. A.2) transmission, accounting for the consumption at both the sender and the receiver:

$$
\begin{aligned}
& T_{T X}\left(P_{T X}+P_{R X, \text { active }}\right)+\left(T_{A C K}+t_{A C K}\right) P_{R X, \text { active }}+T_{A C K} P_{T X}+ \\
& T_{b} P_{R X, \text { active }}+T_{B} P_{R X, \text { off }} \\
& T_{T X}\left(P_{T X}+P_{R X, \text { active }}\right)+T_{A C K, T O} P_{R X, \text { active }}+P_{b} P_{R X, \text { active }}+T_{B} P_{R X, \text { off }}
\end{aligned}
$$

where: $(i) T_{T X}$ is the time to transmit a frame, $P_{T X}$ is the transmission power, $P_{R X, \text { active }}$ and $P_{R X, \text { idle }}$ is the power consumed by an active and idle receiver respectively, (ii) $T_{b}$ and $T_{B}$ are the times the sender spend while backing off with its receiver on and off respectively, (iii) $T_{A C K}$ is the time to transmit/receive an $A C K$, $T_{A C K, T O}$ the time the sender waits to detect the absence of an $A C K$, and $t_{a c k}$ is defined in Table 1. Eqs. A.1, A.2 are used to calculate the total consumed energy needed for the calculation of $J$ in the performance evaluation section. 